\title{
Platelet Concentrate In Lumbar Interbody Cage Fusion: A New Era of Modality Of Fusion
}

\author{
Mohamed Mohi Eldin (D), Ahmed Salah Hassan (D), TarekAhmed Tareef, Mohammad Baraka DiD, Mohamed Gabr, Ahmed H. Omar* \\ Department of Neurosurgery, Faculty of Medicine, Cairo University, Giza, Egypt
}

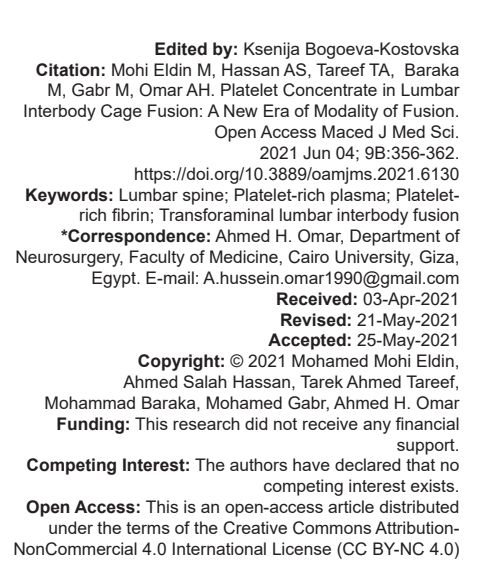

\begin{abstract}
AIM: This was a prospective double-blinded randomized comparative study. Several authors have reported the efficacy of platelets derivatives for spinal union. However, the use of PRP and PRF for bone fusion with TLIF has not been fully explored.

METHODS: Standard open TLIF surgery was performed on 80 patients, randomized in three groups, TLIF and local bone were used in 40 patients (control group) and TLIF, local bone, and PRP were used in 20 patients (PRP group) and PRF was used in 20 patients (PRF group). Radiological parameters were assessed by X-ray after 3 months post-operative to evaluate the position of the screws and cage migration and by CT scans at 6 months and 12 months postoperatively. Patients, surgeons, and post-surgical analysis were blinded.

RESULTS: VAS scores for lower back pain and leg pain were statistically significant between the three groups preoperatively. The VAS scores of back pain improved after 12 months and were statistically significant $(p<0.001)$ and the VAS score of leg pain improved compared with preoperatively in the three groups during the 6-and 12-month follow-up postoperatively, however, the three groups, VAS scores for leg pain were not significantly different. After 1-year follow-up, significantly more patients achieved definite fusion, when implanted platelets derivatives compared with the control group, but with no significance regarding fusion rates.
\end{abstract}

CONCLUSION: We advocated using platelet derivative as a fusion enhancer modality which is cost and time saving It appears to be a complementary step that ensures better outcome for the patients.

\section{Introduction}

Platelet concentrates in spine fusion gained increasing popularity among spine surgeons. They avoid morbidity of bone harvest and promise good union rates without additional device-related adverse events [1], [2].

Platelets concentrates, known as a rich source of growth factors [platelet-derived growth factor, transforming growth factor, insulin-like growth factor, epidermal growth factor, vascular endothelial growth factor, and fibroblast growth factor], have recently been used for muscle, tendon, and bone healing [3], [4], [5], autologous plateletrich fibrin (PRF) and platelet-rich plasma (PRP) have been used also for treating discogenic back pain and sacroiliac joint pain with very promising results [6], [7].

The PRP and PRF represent an evolution of technique that can improve bone union rate and after PLF surgery and significantly shorten the period if sufficiently high concentrations of platelets and bone union-related growth factors are used. Patients were operated with posterolateral fusion combined with PRP showed a higher fusion rate, greater fusion mass, and more rapid bone union compared to control group [8].
The purpose of the current study was to assess the effect of platelets concentrates (PRF and PRP) on the fusion rates when combined with autologous bone graft in trans foraminal lumbar interbody (TLIF) fusion.

\section{Patients and Methods}

This study was approved by ethical committee of neurosurgery department in with IRB approval in October 2020.

A single-center prospective randomized controlled comparative study of 80 patients with lumbar instability underwent transforaminal lumbar interbody cage fusion (TLIF) for single-level pathology was conducted. Approval from the local ethical committee and institutional research board approval was obtained for the study. Randomization was by sealed envelope with a 1:1 ratio opened just before surgery. Indications for surgery were both lytic and degenerative spondylolisthesis, disc degeneration not responding to conservative treatment modalities for at least 6 weeks. The study was restricted to patients with single-level disc pathology. 
Patients with disc pathology at adjacent levels and patients undergoing multilevel surgery were excluded from the study. We excluded patients with a history of previous spinal surgery, severe osteoporosis, systemic disease, malignancy, and chronic use of steroids. A full informed consent was obtained from each patient. All procedures were performed by the same surgeons, during the same period from January 2018 and January 2020, using similar techniques, implants, and similar pedicle screw and rod systems.

Personal history including name, age, and sex, symptomatology including back pain, lower limb pain, and claudicating pain. Pain was analyzed according to severity using the visual analog scale (VAS) and functional disability was assessed using the pre-operative radiological evaluation included $\mathrm{X}$-ray LSS dynamic views and MRI LSS. The patients were divided into three groups, Group (1): Patients operated by conventional lumbar interbody cage fusion with local bone graft only without any platelet's derivatives, Group (2): Patients operated by conventional lumbar interbody cage fusion with addition of platelets concentrates PRF, and Group (3): Patients operated by conventional lumbar interbody cage fusion with addition of platelets concentrates PRP.

\section{Technique of I-PRF preparation}

For each participant, $10 \mathrm{ml}$ peripheral blood was collected under complete aseptic conditions using $10 \mathrm{ml}$ tubes without any additive in three sterile Vacutainer. The tubes were placed in a horizontal centrifuge, with a tube filled with water to maintain the balance during centrifuging for 2 min at $3300 \mathrm{rpm}$ using a standard electronically controlled bench-top centrifuge, this leads to the separation of PRF on top of the remaining blood cells (I-PRF) and the remaining blood materials below. For a collection of the PRF, the tubes were opened carefully, to avoid homogenization of the material, and PRF was aspirated using a $20 \mathrm{ml}$ syringe, with an $18 \mathrm{G}$ hypodermic needle [6].

\section{Technique for I-PRP preparation}

A simple, commercially available technique for the extraction of PRF using $12 \mathrm{ml}$ tubes with anticoagulant citrate dextrose solution (ACD-A). The blood used for preparing the I-PRP was withdrawn from the patients after introducing the needles. The sample is gently agitated to mix the anticoagulant thoroughly with the blood. After collecting six tubes, they were placed in the horizontal centrifuge, for $6 \mathrm{~min}$ at 1000 rpm using a standard electronically controlled bench-top centrifuge. After termination of this process, it is possible to observe an orange color area in the tube (i-PRP) and the remaining blood materials below. The tubes then were opened carefully, to avoid homogenization of the material. We collected at least
$10 \mathrm{ml}$ of i-PRP from the tubes using a $20 \mathrm{ml}$ syringe [6] (Figure 1).

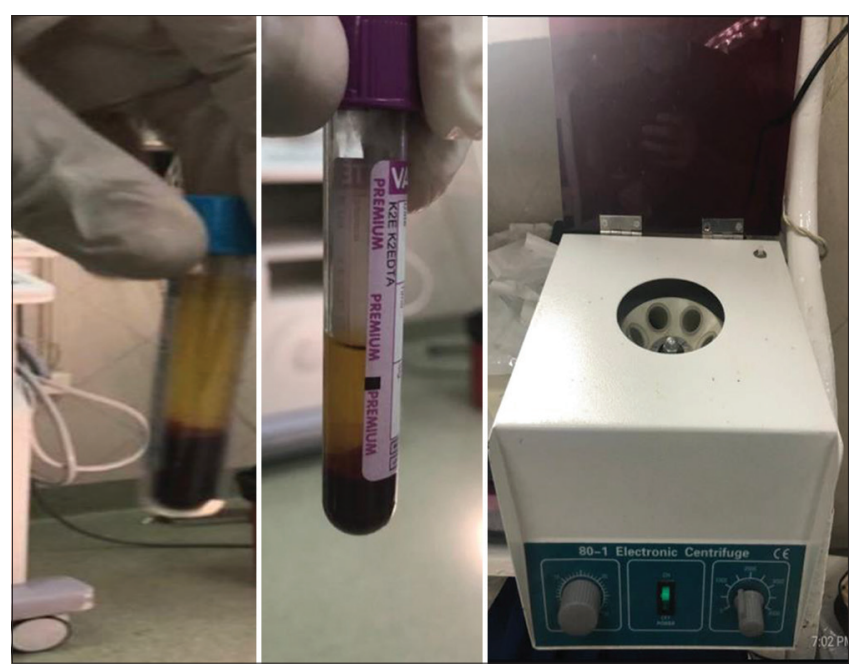

Figure 1: Prepared injectable PRF, PRP, and the electronic centrifuge

\section{Surgical technique}

Conventional transforaminal lumbar interbody cage (TLIF) was performed using pedicle screws and local bone graft with no additional osteoconductive products was added for the spinal fusion. Patients received general anesthesia - operative dose of antibiotic $30 \mathrm{~min}$ before skin incision and were placed in the prone position. A lumbar interbody fusion (TLIF) with posterior pedicle screw fixation was performed through a midline posterior approach. Pedicular screws were placed under fluoroscopic guidance; laminectomy and facetectomy over the affected level and decompression of the nerve root followed by discectomy. The vertebral body endplates were prepared by curetting until point bleeding was seen. Autologous cancellous bone chips were harvested from the removed laminae and facet for interbody fusion. Ten milliliters of PRF were with local bone into the cage in Group (2) and PRP was added in the same way to Group (3). In the control group, the cages were filled with autologous bone in the same way and were implanted without platelets derivatives. The cages were implanted under fluoroscopic guidance, followed by placement of rods with no posterolateral grafts.

\section{Outcome measurements}

\section{Assessment of final bone union rate}

The interbody fusion rates and cage stability were assessed at 6 and 12 months postoperatively using CT. Interbody fusion was graded by the method of Brantigan and Steffee as modified to describe the Fraser definition of locked pseudoarthrosis (BSF scale) [9]. Fusion was defined as at least two observers simultaneously detecting bone fusion (Table 1 and Figure 2). 


\section{Clinical evaluation}

We evaluated the changes in lower back pain, leg pain, preoperatively and at 3,6 , and 12 months postoperatively using the visual analog scale (VAS). Patients were asked to report VAS back and leg pain and preoperatively, and at 3, 6, and 12 months after the surgery. Odom's scale was used for functional outcome assessment at 6- and 12-month follow-up.

Table 1: Brantigan and Steffee as modified to describe the Fraser definition of locked pseudoarthrosis (BSF scale)

\begin{tabular}{ll}
\hline BSF-1 & $\begin{array}{l}\text { Radiographical pseudoarthrosis is indicated by collapse of the } \\
\text { construct, loss of disc height, vertebral slip, displacement of the } \\
\text { carbon cage, broken screws, significant resorption of the bone graft, } \\
\text { or lucency visible around the periphery of the graft or cage }\end{array}$ \\
BSF-2 & $\begin{array}{l}\text { Radiographical locked pseudoarthrosis is indicated by latency visible } \\
\text { in the middle of the cages with solid bone growing into the cage from } \\
\text { each vertebral endplate. } \\
\text { BSF-3 }\end{array}$ \\
& $\begin{array}{l}\text { Radiographical fusion: Bone bridges over at least half of the fusion } \\
\text { area with at least the density originally achieved at surgery }\end{array}$ \\
\hline
\end{tabular}

\section{Statistical analysis}

Data were coded and entered using the Statistical Package for the Social Sciences (SPSS) version 26 (IBM Corp., Armonk, NY, USA). Data were summarized using mean, standard deviation, median, minimum, and maximum in quantitative data and using frequency (count) and relative frequency (percentage) for categorical data. Comparisons between quantitative variables were done using the non-parametric Kruskal-Wallis and Mann-Whitney tests [10]. For comparing categorical data, Chi-square $\left(\chi^{2}\right)$ test was performed. $p<0.05$ was considered statistically significant.

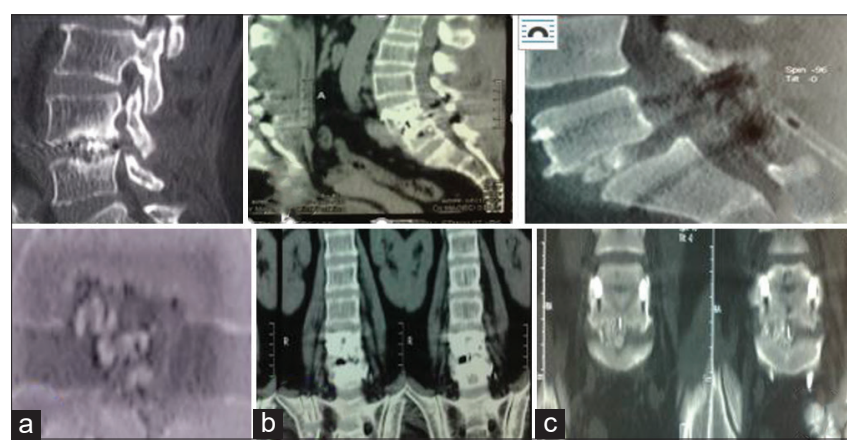

Figure 2: Computed tomography sagittal and coronal illustrating (a) BSF Grade 1 fusion. (b) BSF Grade 2 fusion. (c) BSF Grade 3 fusion

\section{Results}

\section{Demographic}

Patients' demographic data are shown in Table 2. There was no significant difference in the sex, average age, and the level of fusion between the control, PRF, and PRP groups ( $p>0.05)$.

\section{Assessment of bony fusion}

The study flow diagram is schematically represented in Figure 3. All radiological and clinical outcome parameters were assessed preoperatively for each patient. All patients were asked to return for a follow-up CT scan after 6 and 12 months. However, some patients were lost from the radiological follow up. At 6 months after the surgery; we performed CT scans of 33 patients in Group A, 17 patients in Group B, and 16 patients in Group C. In Group A, three patients with fusion Grade 3 after 6 months did not participate in CT scan 12 months after the surgery because their symptoms improved. Furthermore, in Group B, two patients with fusion Grade 2 after 6 months chose not to participate in the follow-up CT scan after 12 months. In Group C, two patients with fusion Grade 3 not receive another CT scan, 12 months after the surgery.

Table 2: Demographic data

\begin{tabular}{|c|c|c|c|c|c|c|c|}
\hline & \multicolumn{2}{|c|}{ Group A $(n=15)$} & \multicolumn{2}{|c|}{ Group B $(n=15)$} & \multicolumn{2}{|c|}{ Group C $(n=15)$} & \multirow[t]{2}{*}{$\mathrm{p}$-value } \\
\hline & $n$ & $\%$ & $n$ & $\%$ & $\mathrm{n}$ & $\%$ & \\
\hline \multicolumn{8}{|l|}{ Sex } \\
\hline Male & 17 & 42.5 & 10 & 50 & 9 & 45 & 0.859 \\
\hline Female & 23 & 57.5 & 10 & 50 & 11 & 55 & \\
\hline Mean age (year) & \multicolumn{2}{|c|}{$45.13 \pm 9.9$} & \multicolumn{2}{|c|}{$47.4 \pm 6.2$} & \multicolumn{2}{|c|}{$44.05 \pm 4.5$} & 0.516 \\
\hline \multicolumn{8}{|l|}{ Level } \\
\hline L4-5 & 36 & 90 & 15 & 75 & 15 & 75 & 1.000 \\
\hline L5-S1 & 4 & 10 & 5 & 25 & 5 & 25 & \\
\hline
\end{tabular}

The radiological outcome of the study was determined by an independent spine radiologist. The fusion grades for each treatment group at the 6-month follow-up and the 12-month follow-up are summarized in Tables 3 and 4 consecutively.

Table 3: Summary of the fusion grades per treatment group, as identified on computed tomography scans performed 6 months after the surgery

\begin{tabular}{|c|c|c|c|c|c|c|c|}
\hline & \multicolumn{2}{|c|}{ Control } & \multicolumn{2}{|l|}{ PRF } & \multicolumn{2}{|l|}{ PRP } & \multirow{2}{*}{ p-value } \\
\hline & Count & $\%$ & Count & $\%$ & Count & $\%$ & \\
\hline \multicolumn{8}{|l|}{ BSF grades } \\
\hline Grade 1 & 15 & 45.5 & 3 & 17.6 & 2 & 12.5 & 0.112 \\
\hline Grade 2 & 13 & 39.4 & 9 & 52.9 & 9 & 56.3 & \\
\hline Grade 3 & 5 & 15.2 & 5 & 29.4 & 5 & 31.3 & \\
\hline
\end{tabular}

At the 6-month follow-up CT scan, $45.5 \%$ of the patients in Group A (control group) had fusion Grade 1, 39.4\% had Grade 2 fusion, and $15.2 \%$ had Grade 3 fusion. In Group B (PRF), 17.6\% of the patients achieved fusion Grade 1. About $52.9 \%$ Grade 2 fusion and $29.4 \%$ Grade 3 fusion. While in Group C (PRP), $12.5 \%$ of the patients achieved fusion Grade 1, 56.3\% Grade 2 fusion, and $31.3 \%$ Grade 3 fusion (Table 3).

Table 4: Summary of the fusion grades per treatment group, as identified on computed tomography scans performed 12 months after the surgery

\begin{tabular}{|c|c|c|c|c|c|c|c|}
\hline & \multicolumn{2}{|l|}{ Control } & \multicolumn{2}{|l|}{ PRF } & \multicolumn{2}{|l|}{ PRP } & \multirow[t]{2}{*}{$\mathrm{p}$-value } \\
\hline & Count & $\%$ & Count & $\%$ & Count & $\%$ & \\
\hline \multicolumn{8}{|c|}{ BSF grades after 12 months } \\
\hline Grade 1 & 2 & 6.7 & 0 & 0.0 & 0 & 0.0 & 0.112 \\
\hline Grade 2 & 14 & 46.7 & 4 & 26.7 & 3 & 21.4 & \\
\hline Grade 3 & 14 & 46.7 & 11 & 73.3 & 11 & 78.6 & \\
\hline
\end{tabular}

In Table 4, we summarize the fusion grades for each cage type at the 12-month follow-up. One year after the surgery, $15.2 \%$ of the patients in Group $A$ (control) achieved definite fusion (Grade 3). In Group B (PRF), 29.4\% of the patients were classified as definite fusion. In Group c (PRP), 31.3\% of the patients 


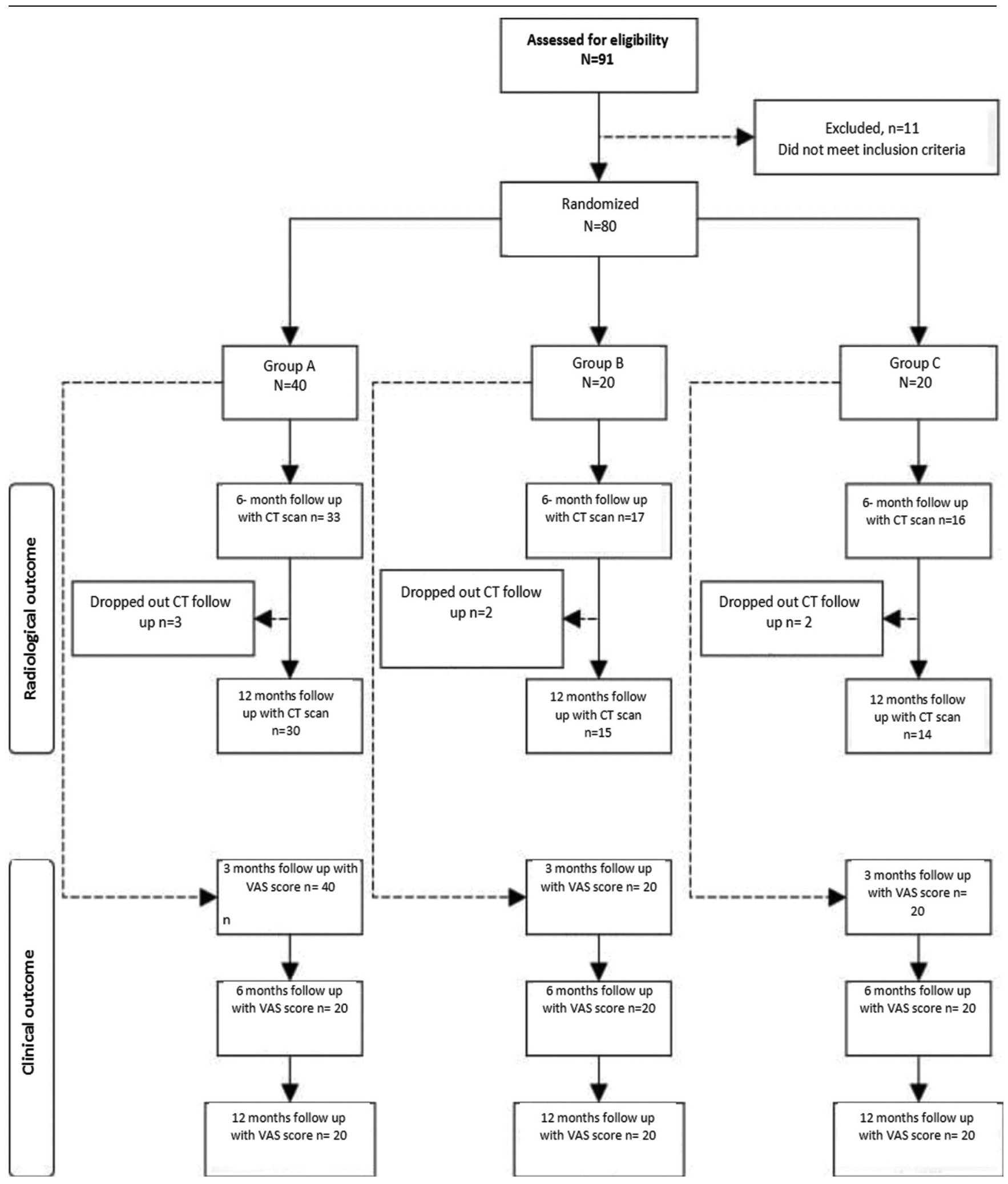

Figure 3: Flowchart during the study showing the number of patients during the follow-up

had definite fusion. Again, significantly more patients achieved definite fusion, when implanted with PEEK cages platelets derivatives compared with the control group, but with no statistical significance.

\section{Visual analog scale pain score}

The VAS scores for lower back pain and leg pain were statistically significant between the three groups preoperatively $(p<0.05)$. The VAS scores of back pain improved after 6-month and after 12-month follow-up, however, it was statistically significant $(p<0.001)$ after 12 months. The VAS score of leg pain improved compared with preoperatively in the three groups during the 6- and 12-month follow-up postoperatively, however, the difference in improvement was not statistically significant $(p>0.05)$ (Tables 5 and 6$)$. 
Table 5: Comparing different groups in terms of VAS back pain

\begin{tabular}{lllll}
\hline Back pain visual analog scale & Group A & Group B & Group C & Significance (p-value) \\
\hline Pre-operative & 6 & 6.5 & 7.5 & $<0.001(\mathrm{~s})$ \\
Post-operative 6 months & 4.5 & 4.35 & 4.40 & 0.832 \\
Post-operative 1 year & 2.2 & 3.05 & 2.05 & $<0.001(\mathrm{~s})$ \\
\hline
\end{tabular}

Table 6: Comparing different groups in terms of VAS leg pain

\begin{tabular}{lllll}
\hline Leg pain visual analog scale & Group A & Group B & Group C & Significance (p-value) \\
\hline Pre-operative & 7.35 & 7.35 & 6.55 & $0.006(\mathrm{~s})$ \\
Post-operative 6 months & 4.07 & 4.25 & 3.15 & 0.832 \\
Post-operative 1 year & 1.83 & 2.20 & 2.05 & 0.338 \\
\hline
\end{tabular}

\section{Clinical outcome scale}

Comparison between control, PRF, and PRP groups regarding assessing the clinical outcome and regaining the activity of daily life at 6 -month and 12-month intervals using the 4-point rating scale (Odom's criteria). Control group had an excellent or good outcome at 6 months interval which was $56.8 \%(30 / 40)$ decreased to $70 \%(28 / 40)$ at 12 months interval while the PRF group had an excellent or good outcome at 6 months interval which was $60 \%(12 / 20)$ decreased to $55 \%(11 / 20)$ at 12 months interval but it is non-significant better outcome the PRP group had an excellent or good outcome at 6 months interval which was $55 \%(11 / 20)$ increased to $65 \%(13 / 20)$ at 12 months interval but it is non-significant better outcome (Table 7).

Table 7: Odom's scale for functional outcome

\begin{tabular}{|c|c|c|c|c|c|c|c|c|}
\hline \multirow[t]{2}{*}{ Odom's criteria } & \multicolumn{4}{|c|}{6 months } & \multicolumn{4}{|c|}{12 months } \\
\hline & Control & PRF & PRP & $p$-value & Control & PRF & PRP & $p$-value \\
\hline Excellent & 9 & 5 & 6 & 0.38 & 10 & 6 & 8 & 0.45 \\
\hline Good & 21 & 7 & 5 & 0.40 & 18 & 5 & 5 & 0.44 \\
\hline Fair & 8 & 4 & 7 & 0.50 & 11 & 8 & 5 & 0.90 \\
\hline Poor & 2 & 4 & 2 & 0.46 & 1 & 1 & 2 & 0.30 \\
\hline
\end{tabular}

\section{Discussion}

Pseudoarthrosis remains a significant problem in spinal fusion, the incidence of which ranges from $5-34 \%$ in various series [11], [12]. The use of PRF and PRP in TLIF surgery is still controversial, Lowery et al. first reported 19 cases of lumbar spinal fixation using platelet concentrates (posterior fusion, $n=15$; anterior intradiscal fusion, $n=4$ ). In five patients, bone union was confirmed at the time of the second surgery, whereas in the remaining 14 patients, bone union was confirmed using radiographic assessment [13].

Meanwhile, Weiner and Walker concluded that the use of platelet concentrates resulted in inferior fusion rates compared with autogenous bone graft alone in single-level PLF using iliac crest bone graft or iliac crest bone graft plus platelet concentrates [14]. Carreon et al. compared 76 consecutive patients who underwent instrumented PLF with autologous iliac crest bone graft mixed with platelet concentrates with a control group who underwent the same surgery with autologous bone graft alone and concluded that platelet gel failed to enhance fusion rate [15].

In contrast, several authors have reported the efficacy of PRP for interbody fusion surgery, Hee et al. [16] compared 23 patients who underwent TLIF with PRP application and a historical cohort (without PRP application) with a minimum of 2-year follow-up was compared. They demonstrated faster fusion but no increase in fusion rates. Jenis LG et al. [17] compared 37 patients who underwent anterior-posterior interbody fusion with iliac crest bone graft or allograft combined with PRP which were evaluated for 2 years; the rate of fusion was $89 \%$ in the PRP group compared with $85 \%$ in the control group, respectively, Elder et al. [18] concluded that platelet gel may be a low cost, low-risk profile, and low complication rate strategy in the future after systematically reviewing all studies regarding PRP and PRF in spinal fusion from January 1990 to September 2014.

We found that the technique of using PRF and PRP was simple and cost effective. The union rate was higher in the PRP group as well as better clinical outcome with no difference in post-operative complications.

The improvement in the back and leg pain score during the follow-up after adding the platelets derivatives to lumbar interbody cage is still controversial. Kubota et al. [19] in a case series of 20 patients operated on TLIF with local bone graft with and without PRP found no significant difference in lower back pain, leg pain, and leg numbness between the two groups at the final follow-up. Sys et al. [20] in their retrospective study of 38 patients who underwent posterior stabilization with autograft addition to PRP and autograft alone in the control group found improvement of VAS in both groups, but the difference was not statistically significant between the groups at the final follow-up.

We utilized that the VAS scores for lower back pain and leg pain were statistically significant between the three groups preoperatively $(p<0.05)$. The three VAS scores improved postoperatively compared with preoperatively in the three groups during the 6-and 12-month follow-up postoperatively, however, the VAS scores were significantly different between the three groups regarding the back pain after 12 months follow-up $(p<0.05)$.

Different preparation methods and timing between PRP activation and implantation may have a role in growth factors concentrations [21]. In our study, the initial platelet count in PRP and PRF ranged between 153 and $355 \times 10^{3} / \mathrm{cm}^{3}$ with a mean value of $217 \pm 55.6 \times 10^{3} / \mathrm{cm}^{3}$. Platelet count was re-estimated after PRP preparation and it was found to be almost double to triple than the initial platelet count. It ranged between 299 and $599 \times 10^{3} / \mathrm{cm}^{3}$ with a mean value of $427 \pm 90 \times 10^{3} / \mathrm{cm}^{3}$. Initial platelet count in PRF patients ranged between 155 and $382 \times 10^{3} / \mathrm{cm}^{3}$ with a mean value of $229 \pm 55.6 \times 10^{3} / \mathrm{cm}^{3}$. It could not be estimated after PRF preparation. 


\section{Limitation of study}

The present study has several limitations. First, relatively small sample size and the short follow-up period. Second, the amount and quality of the local autograft were not uniform between patients, but this limitation existed in both treatment groups and we did not compare other types of bone substitutes such as artificial bone particles and iliac bone graft. Finally, we did not include any comorbidities in our study such as diabetes or osteoporosis.

\section{Conclusion}

The use of growth factors in spinal fusion is a promising area, it is proved in general for promoting bony union. Our study concluded that both PRF and PRP increase the rate of fusion when combined with lumbar interbody with improvement of the clinical course, however, these results were not statistically significant due to the relatively small sample size with short-term follow-up. Enhanced fusion rates mean that patients recover faster and have less need of orthosis protection, less incidence of pseudoarthrosis, and rapid return to daily life activities. The technique for the preparation of platelets concentrates is widely available and low cost make which makes it easy and cheap to use and may be a promising technique for spinal fusion.

\section{Conflicts of Interest}

The authors declare that there are no conflicts of interest regarding the publication of this paper.

\section{Informed Consent}

Informed consent was obtained from all individual participants included in this study.

\section{References}

1. Hustedt JW, Blizzard DJ. The controversy surrounding bone morphogenetic proteins in the spine: A review of current research. Yale J Biol Med. 2014;87(4):549-61. PMid:25506287

2. Landi A, Tarantino R, Marotta N, Ruggeri AG, Domenicucci M, Giudice $\mathrm{L}$, et al. The use of platelet gel in postero-lateral fusion: Preliminary results in a series of 14 cases. Eur Spine J. 2011;20(Suppl 1):S61-7. https://doi.org/10.1007/ s00586-011-1760-3

PMid:21416280

3. Malhotra A, Pelletier MH, Yu Y, Walsh WR. Can plateletrich plasma (PRP) improve bone healing? A comparison between the theory and experimental outcomes. Arch Orthop Trauma Surg. 2013;133(2):153-65. https://doi.org/10.1007/ s00402-012-1641-1

PMid:23197184

4. Sadoghi $\mathrm{P}$, Lohberger $\mathrm{B}$, Aigner $\mathrm{B}$, Kaltenegger $\mathrm{H}$, Friesenbichler $\mathrm{J}$, Wolf $\mathrm{M}$, et al. Effect of platelet-rich plasma on the biologic activity of the human rotator-cuff fibroblasts: A controlled in vitro study. J Orthop Res. 2013;31(8):1249-53. https://doi.org/10.1002/jor.22360

PMid:23568511

5. Vavken J, Vavken $\mathrm{P}$, Mameghani A, Camathias C Schaeren S. Platelet concentrates in spine fusion: Metaanalysis of union rates and complications in controlled trials. Eur Spine J. 2016;25(5):1474-83. https://doi.org/10.1007/ s00586-015-4193-6

PMid:26298478

6. Eldin MM, Hassan AS, Baraka M, Khorshied M. Intradisca injection of autologous platelet-rich fibrin versus platelet-rich plasma in discogenic lumbar pain: An applied comparative study. J Orthop Trauma Surg Relat Res. 2020;15:(1).https://doi. org/10.3233/bmr-181366

7. Mohi Eldin M, Sorour OO, Hassan ASA, Baraka M, Ahmed MF Percutaneous injection of autologous platelet-rich fibrin versus platelet-rich plasma in sacroiliac joint dysfunction: An applied comparative study. J Back Musculoskelet Rehabil. 2019;32(3):511-8. https://doi.org/10.3233/bmr-181366 PMid:30958332

8. Han B, Woodell-May J, Ponticiello M, Yang Z, Nimni M The effect of thrombin activation of platelet-rich plasma on demineralized bone matrix osteoinductivity. J Bone Joint Surg Am. 2009;91(6):1459-70. https://doi.org/10.2106/jbjs.h.00246 PMid:19487525

9. Brantigan JW, Steffee AD. A carbon fiber implant to aid interbody lumbar fusion. Two-year clinical results in the first 26 patients. Spine (Phila Pa 1976). 1993;18(14):2106-17. https:// doi.org/10.1097/00007632-199310001-00030

PMid:8272967

10. Bagdonavičius V, Kruopis J, Nikulin MS. Nonparametric Tests for Complete Data. New Delhi: Indian Society for Technical Education; 2011.

11. Brown CW, Orme TJ, Richardson HD. The rate of pseudarthrosis (Surgical nonunion) in patients who are smokers and patients who are nonsmokers: A comparison study. Spine (Phila Pa 1976). 1986;11(9):942-3. https://doi. org/10.1097/00007632-198611000-00015

PMid:3824072

12. Bydon M, De La Garza-Ramos R, Abt NB, Gokaslan ZL, Wolinsky JP, Sciubba DM, et al. Impact of smoking on complication and pseudarthrosis rates after single- and 2-level posterolateral fusion of the lumbar spine. Spine (Phila $\mathrm{Pa}$ 1976). 2014;39(21):1765-70. https://doi.org/10.1097/ brs.0000000000000527

PMid:25054650

13. Lowery GL, Kulkarni S, Pennisi AE. Use of autologous growth factors in lumbar spinal fusion. Bone. 1999;25(2 Suppl):47S-50. https://doi.org/10.1016/s8756-3282(99)00132-5 PMid: 10458274

14. Weiner BK, Walker M. Efficacy of Autologous Growth Factors in Lumbar Intertransverse Fusions. Spine (Phila 
Pa 1976). 2003;28(17):1968-70. https://doi.org/10.1097/01. brs.0000083141.02027.48

PMid: 12973143

15. Carreon LY, Glassman SD, Anekstein Y, Puno RM. Platelet gel (AGF) fails to increase fusion rates in instrumented posterolateral fusions. Spine (Phila Pa 1976). 2005;30(9):2436. https://doi.org/10.1097/01.brs.0000160846.85397.44 PMid: 15864142

16. Hee HT, Majd ME, Holt RT, Myers L. Do autologous growth factors enhance transforaminal lumbar interbody fusion? Eur Spine J. 2003;12(4):400-7. https://doi.org/10.1007/s00586-003-0548-5 PMid:12761669

17. Jenis LG, Blanco RJ, Kwon B. A prospective study of Autologous Growth Factors (AGF) in lumbar interbody fusion. Spine J. 2006;6(1):14-20. https://doi.org/10.1016/j.spinee.2005.08.014 PMid: 16413442

18. Elder BD, Holmes C, Goodwin CR, Lo SF, Puvanesarajah V, Kosztowski TA, et al. A systematic assessment of the use of platelet-rich plasma in spinal fusion. Ann Biomed
Eng. 2015;43(5):1057-70. https://doi.org/10.1007/ s10439-015-1300-0

PMid:25794962

19. Kubota G, Kamoda H, Orita S, Inage K, Ito M, Yamashita M, et al. Efficacy of platelet-rich plasma for bone fusion in transforaminal lumbar interbody fusion. Asian Spine J. 2018;12(1):112-8. https://doi.org/10.4184/asj.2018.12.1.112

PMid:29503690

20. Sys J, Weyler J, Van Der Zijden T, Parizel P, Michielsen J. Platelet-rich plasma in mono-segmental posterior lumbar interbody fusion. Eur Spine J. 2011;20(10):1650-7. https://doi. org/10.1007/s00586-011-1897-0

PMid:21744284

21. Kubota G, Kamoda H, Orita S, Yamauchi K, Sakuma $Y$, Oikawa $Y$, et al. Platelet-rich plasma enhances bone union in posterolateral lumbar fusion: A prospective randomized controlled trial. Spine J. 2019;19(2):e34-40. https://doi. org/10.1016/j.spinee.2017.07.167

PMid:28735763 